\title{
Obscured accretion from AGN surveys
}

\author{
Cristian Vignali ${ }^{1,2}$ \\ ${ }^{1}$ Dipartimento di Fisica e Astronomia, Università di Bologna, \\ Viale Berti Pichat 6/2, 40127 Bologna, Italy \\ ${ }^{2}$ INAF-Osservatorio Astronomico di Bologna, Via Ranzani 1, 40127 Bologna, Italy \\ email: cristian.vignali@unibo.it
}

\begin{abstract}
Recent models of super-massive black hole (SMBH) and host galaxy joint evolution predict the presence of a key phase where accretion, traced by obscured Active Galactic Nuclei (AGN) emission, is coupled with powerful star formation. Then feedback processes likely selfregulate the SMBH growth and quench the star-formation activity. AGN in this important evolutionary phase have been revealed in the last decade via surveys at different wavelengths. On the one hand, moderate-to-deep X-ray surveys have allowed a systematic search for heavily obscured AGN, up to very high redshifts $(\mathrm{z} \approx 5)$. On the other hand, infrared/optical surveys have been invaluable in offering complementary methods to select obscured AGN also in cases where the nuclear X-ray emission below $10 \mathrm{keV}$ is largely hidden to our view. In this review I will present my personal perspective of the field of obscured accretion from AGN surveys.
\end{abstract}

\section{Introduction}

One of the main science goals of modern observational cosmology is devoted to understand how galaxies and SMBHs at their centers grow together. Their close link leaves imprints in several relations observed in the local Universe between the mass of the black holes and the properties of the host galaxies (e.g., their velocity dispersion; Gebhardt et al. 2000; Ferrarese \& Merritt 2000). The emerging picture is that AGN are the key to understand the nature of such close connection, since the mass function of local SMBHs can be reasonably explained by the growth of seed black holes (whatever the origin of such seeds is) during AGN phases (e.g., Soltan 1982; Marconi et al. 2004).

The entire picture, related to the so-called AGN-galaxy co-evolution scenario, has been presented in many works over the last decade, and has been perfectly synthesized in Fig. 1 of Hopkins et al. (2008), along the path traced by the original suggestion of Sanders et al. 1988 (see also Sanders \& Mirabel 1996). Concisely, current quasar/host galaxy co-evolution models predict the existence of a dust-enshrouded phase associated with rapid SMBH growth and active star formation, largely triggered by multiple galaxy mergers and encounters (e.g., Silk \& Rees 1998; Di Matteo et al. 2005; Menci et al. 2008; Zubovas \& King 2012; Lamastra et al. 2013). This phase is likely associated to obscured AGN growth in strongly star-forming (sub-millimeter) galaxies (e.g., Alexander et al. 2005). Finally, massive quasar-driven outflows blow away most of the cold gas reservoir, creating a population of "red-and-dead" gas-poor elliptical galaxies (e.g., Cattaneo et al. 2009).

Support to this scenario comes from observations of wide-angle molecular outflows extending few kpc from the nucleus in some quasars hosted in ultra-luminous infrared galaxies; these systems, typically characterized by mass loss rates much larger than the ongoing star-formation rate (e.g., Feruglio et al. 2010; Sturm et al. 2011; Rupke \& Veilleux 2013; Cicone et al. 2014), are observed up to very high redshifts (Maiolino et al. 2012; Borguet et al. 2013). Similarly, observations of powerful outflows in neutral and ionized gas have also been collected over the past few years (e.g., Nesvadba et al. 2008; Alexander 
et al. 2010; Harrison et al. 2012). This feedback process ascribed to quasars is most certainly related to radiation-driven winds and is often invoked to explain why SMBHs and galaxies stop growing at a certain phase of their life; for a more comprehensive discussion on this issue, see the review by C. M. Harrison (this Volume). Evidences for ultra-fast outflows (with velocities typically up to 0.1-0.4c) have been recently observed in X-rays in a sizable sample of AGN, both in the local Universe (e.g., Tombesi et al. 2010, 2011, 2012; Gofford et al. 2013; Reeves et al. 2003) and at high redshift (e.g., Chartas et al. 2002, 2007; Saez et al. 2009). The connection between molecular and highly ionized gas is, however, from from being assessed, and will constitute undoubtedly one of the prime science goals of the coming years using $A L M A$ and IRAM facilities at long wavelengths and Chandra and XMM-Newton in the X-ray domain.

According to the scenario described above, the main trigger mechanism of $\mathrm{BH}$ accretion and growth is ascribed to galaxy mergers and interactions, at least in the most luminous and massive systems. Most of their mass is assembled in short periods $(\approx 10-100 \mathrm{Myr})$ of "bursting" nuclear and star-forming activity, while the bulk of galaxies and SMBHs grow their mass in a secular (i.e., "smooth") mode over timescales of Gyrs (e.g., Daddi et al. 2007a; Hickox et al. 2009). This picture has recently been confirmed by Herschel surveys, showing a distinction between the bulk of galaxies growing quietly (in the socalled "main sequence") and the minority of the galaxy population whose growth happens mostly during events of mergers of gas-rich galaxies in the so-called "starburst mode" (e.g., Elbaz et al. 2011; Rodighiero et al. 2011; see also Rosario et al. 2013).

As a natural consequence of the merger scenario, a key phase in the AGN and galaxy life is when large amounts of gas are funneled to the center, thus inducing both obscured accretion and star formation (e.g., Treister et al. 2010). Significant efforts have been made recently to search for and characterize, as much as possible, the most heavily obscured AGN and quasars, dubbed Compton thick, characterized by column densities above $1.5 \times 10^{24} \mathrm{~cm}^{-2}$ (see Comastri 2004 for a review); such absorbers strongly limit the possibility for these sources of being detected at energies below $10 \mathrm{keV}$ (where sensitive $\mathrm{X}$-ray imaging instruments are currently operative). Therefore, in order to provide a census as complete as possible of this source population, a multi-wavelength synergistic approach is needed.

In this review I will focus on some aspects and methods of investigation that I think are important in the quest for heavily obscured AGN. As such, this proceeding is not meant to provide an exhaustive view of this topic. Further and, possibly, alternative approaches in this research field and consequences for AGN synthesis models of the X-ray background (XRB) are addressed by other authors in this Volume (e.g., A. Barger, A. Del Moro, S. Juneau, A. Levenson, S. Mateos, L. Spinoglio, D. Stern, E. Treister, Y. Ueda).

\section{Searching for heavily obscured AGN}

The problem of finding heavily obscured AGN and quasars can be tackled following various prescriptions and adopting different approaches. The bad news is that there is no way to obtain a complete census of this AGN population either using single-band observations or a unique selection method/criterion. The good news is that the multiwavelength observing campaigns which characterize most of the current surveys offer a unique possibility to detect the most obscured AGN, up to very high redshifts. Adopting several selection criteria and keeping in mind the observational biases intrinsic to each detection band are what we need in the future to infer the demographics of these elusive AGN and use them to provide "boundary" conditions and useful constraints to AGN/galaxy co-evolution models. 


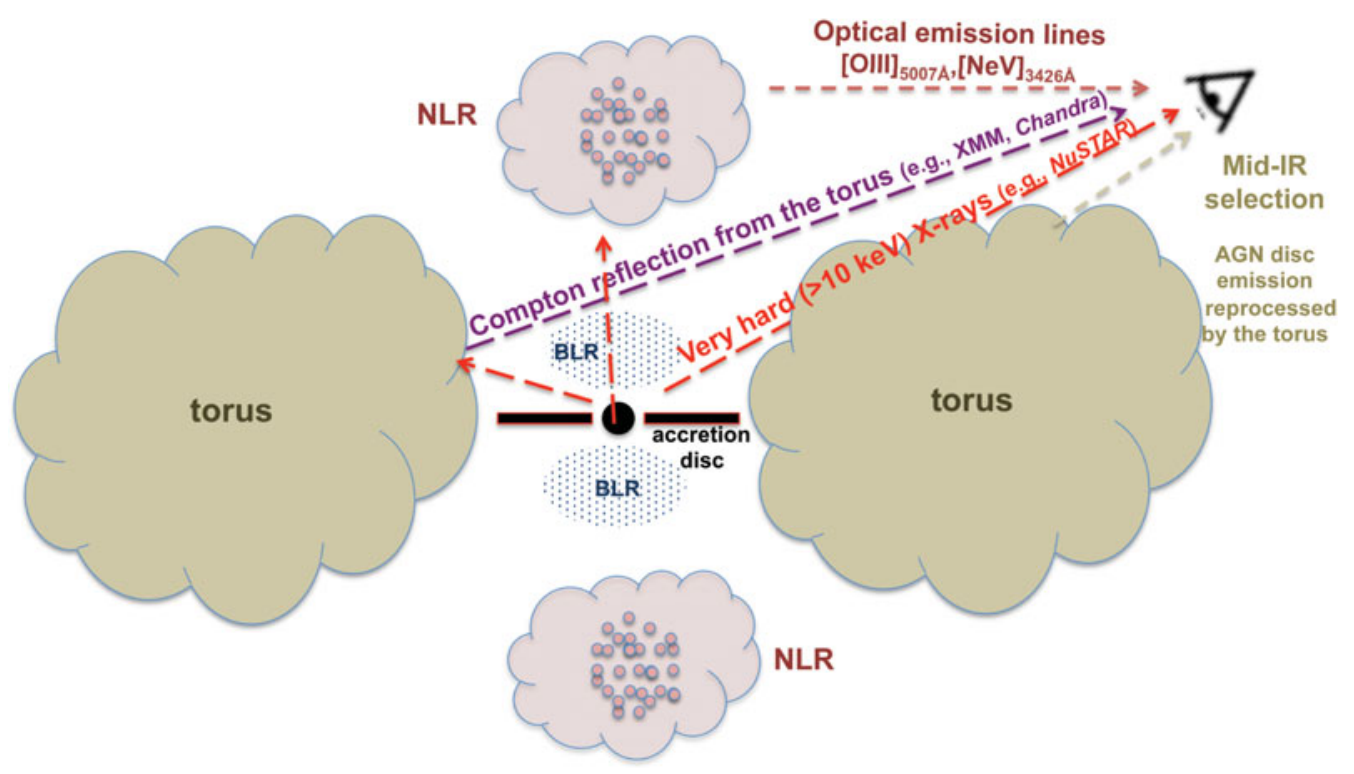

Figure 1. Schematic view of AGN (not in scale). Emphasis is given to the emission components which, at different wavelengths, allow for the detection of obscured AGN. BLR and NLR stand for broad-line region and narrow-line region, respectively.

In the following, I will try to elucidate some detection techniques adopted to find obscured AGN, which are schematized in Fig. 1. In particular, I am referring to methods related to X-ray (§2.1), mid-infrared (mid-IR; $§ 2.2)$ and optical selection (§2.3).

\subsection{Hard X-ray surveys}

According to the unified model for AGN (Antonucci 1993), the X-ray emission, once it intercepts the obscuring material (i.e., the torus; see Fig. 1), can be profoundly depressed in the X-ray band. In particular, if the optical depth for Compton scattering $\left(\tau=N_{H} \times\right.$ $\left.\sigma_{T}\right)$ does not exceed values of the order of " $a$ few", X-ray photons with energies higher than 10-15 keV are able to penetrate the obscuring material and reach the observer. For higher values of $\tau$, the entire X-ray spectrum is depressed by Compton down-scattering and the X-ray photons are effectively trapped by the obscuring material irrespective of their energy. The former class of sources (mildly Compton thick) can be efficiently detected by X-ray instruments above $10 \mathrm{keV}$, while for the latter (heavily Compton thick) their nature may be inferred through indirect arguments, such as the presence of a strong iron $\mathrm{K} \alpha$ emission line over a flat reflected continuum. Mildly Comptonthick AGN are the most promising candidates to explain the residual (i.e., not resolved yet) spectrum of the cosmic XRB at its $30 \mathrm{keV}$ peak (e.g., Worsley et al. 2005; Gilli et al. 2007; Ballantyne 2009; Treister et al. 2009; Moretti et al. 2012; Shi et al. 2013) but only a handful of them are known (i.e., have been classified as such beyond any reasonable doubt) outside the local Universe (e.g., Iwasawa et al. 2005). An unbiased census of extremely obscured AGN would require to survey the hard X-ray above $10 \mathrm{keV}$ with a fairly good sensitivity. A step forward in this direction is being provided by the Swift/BAT and Integral/IBIS surveys (e.g., Tueller et al. 2008; Beckmann et al. 2009; Vasudevan et al. 2013), which have covered a large portion of the sky though limited 
to relatively bright X-ray fluxes $\left(\approx 10^{-11} \mathrm{erg} \mathrm{cm}^{-2} \mathrm{~s}^{-1}\right)$, hence to low redshifts, and have resolved less than $10 \%$ of the XRB. The spectral characterization of the heavily obscured AGN discovered in these shallow hard X-ray surveys often required follow-up observations with the more sensitive instruments onboard Chandra, XMM-Newton and Suzaku (e.g., Eguchi et al. 2009; Comastri et al. 2010; Winter et al. 2010; Severgnini et al. 2011; Burlon et al. 2011); this approach led to an estimate of a fraction of $\approx 10-20 \%$ of Compton-thick AGN among hard X-ray selected samples (e.g., Malizia et al. 2009; Burlon et al. 2011; Vasudevan et al. 2013). Data from the NuSTAR satellite, having imaging capabilities up to $\approx 80 \mathrm{keV}$, can shed new light on this topic at sensitivities more than a factor 100 better than those achieved by Integral and Swift (Alexander et al. 2013).

Deep X-ray surveys with sensitive imaging instruments (Chandra and XMM-Newton) can push the detection of Compton-thick AGN at considerably higher redshifts (e.g., $z=4.75$, Gilli et al. 2011). Indications of Compton-thick material in AGN and quasars have been found by many authors, often coupled to powerful star formation (from few hundred to $\approx 1000 \mathrm{M}_{\odot} / \mathrm{yr}$ ), mostly using the deep exposures in the Chandra Deep FieldSouth (CDF-S) provided by both Chandra (currently $4 \mathrm{Ms}$ - Xue et al. 2011 - close to be extended to $7 \mathrm{Ms}$ ) and XMM-Newton ( $\approx 3 \mathrm{Ms}$; Ranalli et al. 2013); see, e.g., Tozzi et al. (2006); Georgantopoulos et al. (2009, 2013); Comastri et al. (2011); Feruglio et al. (2011); Brightman \& Ueda (2012); Vito et al. (2013). For a significant fraction of X-ray sources found in deep fields, the signal-to-noise ratio of the spectra is limited and does not allow for a proper characterization of the source spectral complexities. Further constraints on the obscured AGN population may be derived using X-ray stacking techniques which take benefit of the good spatial resolution (primarily offered by Chandra) and allow exploration of considerably deeper X-ray fluxes (e.g., Xue et al. 2012). However, even the deepest X-ray exposures currently available miss a significant number of very obscured AGN, hence a not negligible fraction of the accretion power in the Universe.

Another interesting result which is emerging from deep X-ray surveys is related to the increasing fraction of heavily obscured quasars from $\mathrm{z}=0$ to $\mathrm{z} \approx 3-4$; a similar trend is apparently not observed in lower luminosity AGN (Iwasawa et al. 2012; Vito et al. 2013). Since the fraction of AGN in mergers seems to increase with the bolometric luminosity (Treister et al. 2012), we may expect that at high redshift, when the merger rate was higher, a larger gas fraction (producing obscuration) was available in galaxies. The planned extension of Chandra observations in the CDF-S, coupled to very deep infrared data (e.g., CANDELS), will hopefully allow us to explore this hypothesis at very high redshifts in a couple of years.

\subsection{Mid-infrared selection}

The mid-IR regime offers much potential for discovery of heavily obscured AGN, since any primary AGN continuum (i.e., disc emission) that is absorbed must ultimately come out at these wavelengths after being thermally reprocessed by the torus (see Fig. 1). Thus, sources with weak emission in the optical band (because of extinction) and relatively bright mid-IR emission can be counted as heavily obscured AGN candidates, unless a significant contribution in the mid-IR comes from star-formation processes (PAH features and continuum emission). This probably "basic" high mid-IR/optical flux-ratio selection method found support in many works in the era of the Spitzer observatory (e.g., Martínez-Sansigre et al. 2005; Houck et al. 2005; Weedman et al. 2006), and allowed Dey et al. (2008) to define a new class of sources at $z \approx 2$, the dusty obscured galaxies (DOGs), having $F_{24} \mu \mathrm{m} / F_{R}>1000$. Among these, we may expect some of the most obscured AGN, especially if a selection at $F_{24} \mu \mathrm{m}>1 \mathrm{mJy}$ is adopted to limit 
the contamination from star-forming galaxies (e.g., Sacchi et al. 2009). This selection is different from those allowed by the widely adopted mid-IR color-color diagrams (e.g., Lacy et al. 2004; Stern et al. 2005; see also Donley et al. 2012), where separating the most heavily obscured AGN from the remaining source populations is not a trivial job (e.g., Castelló-Mor et al. 2013). However, only X-ray data have been able to provide the smoking gun of the truly Compton-thick nature for a fraction of the high mid-IR/optical flux-ratio sources (e.g., Polletta et al. 2006; Lanzuisi et al. 2009; Georgantopoulos et al. 2011; see also Severgnini et al. 2012). Furthermore, X-ray stacking analyses have allowed to place observational constraints, for the first time, to the space density of Comptonthick AGN at high redshifts $(z \approx 2-3$; Daddi et al. 2007b; Fiore et al. 2008, 2009; Bauer et al. 2010; Alexander et al. 2011; but see also Georgakakis et al. 2010).

Extension of the mid-IR search for heavily obscured AGN is within the capabilities offered by WISE, as shown by Mateos et al. (2013) and D. Stern (this Volume).

\subsection{Optical selection}

The selection of obscured AGN using optical spectroscopy proceeds primarily through the detection of high-ionization emission lines, e.g., [O III] $5007 \AA$ and [Ne v] $3426 \AA$. These lines, being produced in the narrow-line region (NLR), do not suffer from extinction from the torus and are considered good proxies of the nuclear intrinsic power (see Fig. 1). Applying the relation between [O III] and 2-10 keV emission (e.g., Mulchaey et al. 1994; Heckman et al. 2005; Panessa et al. 2006) to the sample of narrow-line AGN from the Sloan Digital Sky Survey of Zakamska et al. (2003) led some authors (e.g., Vignali et al. 2006, 2010; Ptak et al. 2006) to the discovery of about a dozen of Compton-thick AGN candidates. These studies allowed a first estimate of the space density of this obscured AGN population at $z \approx 0.3-0.8$. According to Gilli et al. (2007, 2013) XRB models, the fraction of XRB emission at $20 \mathrm{keV}$ produced by Compton-thick AGN and still "missing" has a peak at $z \approx 0.7$ and is mostly due to Seyfert-like objects, with intrinsic 2-10 keV luminosity below $10^{44} \mathrm{erg} \mathrm{s}^{-1}$. Moving these investigations to slightly higher redshifts requires the use of the $[\mathrm{Ne} \mathrm{V}]$ emission line, which has the advantage of being an unambiguous marker of AGN (with a ionization potential of $97 \mathrm{eV}$ vs. $54 \mathrm{eV}$ of [O III]) but is $\approx 9$ times weaker than [O III] and suffers from stronger extinction. Calibrating the X-ray-to-[Ne V] luminosity ratio on a sample of local AGN, Gilli et al. (2010) show that values $<15$ are highly indicative of Compton-thick obscuration. How effective this line is in finding Compton-thick AGN has been recently confirmed by Mignoli et al. (2013), where narrow-line AGN were selected from the zCOSMOS survey and X-ray coverage was provided by Chandra (Vignali et al., in preparation). About $40 \%$ of the original $\approx 70$ candidates are consistent with being Compton thick (in line with Gilli et al. 2007 model). We note, however, that optical spectroscopy, because of extinction within the NLR, is far from offering a complete census of obscured AGN (see §3.3 of Mignoli et al. 2013). Further insights into the properties of these [ $\mathrm{Ne} \mathrm{v}$ ]-selected Compton-thick AGN will come out by using their mid-IR emission as another proxy of the nuclear emission (e.g., Gandhi et al. 2009) to be compared to the observed X-ray luminosity.

\section{Conclusions}

Obscured AGN growth is a key phase in SMBH/galaxy co-evolution models. As the census of such objects is difficult, especially at high redshifts, a multi-wavelength synergistic approach is needed, requiring deep X-ray exposure, mid-IR data and, possibly, optical/near-IR spectroscopy. Whatever the adopted selection method is, X-rays represent a powerful and fundamental probe through direct X-ray spectroscopy and stacking analysis. 


\section{Acknowledgements}

I would like to thank the organizers of this Symposium and all of my close collaborators for the many enlightening discussions.

\section{References}

Alexander, D. M., Smail, I., Bauer, F. E., et al. 2005, Nature, 434, 738

Alexander, D. M., Swinbank, A. M., Smail, I., et al. 2010, MNRAS, 402, 2211

Alexander, D. M., Bauer, F. E., Brandt, W. N., et al. 2011, ApJ, 738, 44

Alexander, D. M., Stern, D., Del Moro, A., et al. 2013, ApJ, 773, 125

Antonucci, R. 1993, ARAESA, 31, 473

Ballantyne, D. R. 2009, ApJ, 698, 1033

Bauer, F. E., Yan, L., Sajina, A., \& Alexander, D. M. 2010, ApJ, 710, 212

Beckmann, V., Soldi, S., Ricci, C., et al. 2009, A\& A, 505, 417

Borguet, B. C. J., Arav, N., Edmonds, D., Chamberlain, C., \& Benn, C. 2013, ApJ, 762, 49

Brightman, M. \& Ueda, Y. 2012, MNRAS, 423, 702

Burlon, D., Ajello, M., Greiner, J., et al. 2011, ApJ, 728, 58

Castelló-Mor, N., Carrera, F. J., Alonso-Herrero, A., et al. 2013, A $\& A$, 556, A114

Cattaneo, A., Faber, S. M., Binney, J., et al. 2009, Nature, 460, 213

Chartas, G., Brandt, W. N., Gallagher, S. C., \& Garmire, G. P. 2002, ApJ, 579, 169

Chartas, G., Eracleous, M., Dai, X., Agol, E., \& Gallagher, S. 2007, ApJ, 661, 678

Cicone, C., Maiolino, R., Sturm, E., et al. 2014, A\& A, 562, A21

Comastri, A. 2004, in: A. J. Barger (eds.), Supermassive Black Holes in the Distant Universe, (Astrophysics and Space Science Library: Kluwer), 308, 245

Comastri, A., Iwasawa, K., Gilli, R., et al. 2010, ApJ, 717, 787

Comastri, A., Ranalli, P., Iwasawa, K., et al. 2011, A\&广A, 526, L9

Daddi, E., et al. 2007a, ApJ, 670, 156

Daddi, E., et al. 2007b, ApJ, 670, 173

Dey, A., Soifer, B. T., Desai, V., et al. 2008, ApJ, 677, 943

Di Matteo, T., Springel, V., \& Hernquist, L. 2005, Nature, 433, 604

Donley, J. L., Koekemoer, A. M., Brusa, M., et al. 2012, ApJ, 748, 142

Eguchi, S., Ueda, Y., Terashima, Y., Mushotzky, R., \& Tueller, J. 2009, ApJ, 696, 1657

Elbaz, D., Dickinson, M., Hwang, H. S., et al. 2011, A\&\&A, 533, A119

Ferrarese, L. \& Merritt, D. 2000, ApJ (Letters), 539, L9

Feruglio, C., Maiolino, R., Piconcelli, E., et al. 2010, A\& A (Letters), 518, L155

Feruglio, C., Daddi, E., Fiore, F., et al. 2011, ApJ (Letters), 729, L4

Fiore, F., Grazian, A., Santini, P., et al. 2008, ApJ, 672, 94

Fiore, F., Puccetti, S., Brusa, M., et al. 2009, ApJ, 693, 447

Gandhi, P., Horst, H., Smette, A., et al. 2009, A\&A, 502, 457

Gebhardt, K., Bender, R., Bower, G., et al. 2000, ApJ (Letters), 539, L13

Georgakakis, A., Rowan-Robinson, M., Nandra, K., et al. 2010, MNRAS, 406, 420

Georgantopoulos, I., Akylas, A., Georgakakis, A., \& Rowan-Robinson, M. 2009, A\&A, 507, 747

Georgantopoulos, I., Rovilos, E., Akylas, A., et al. 2011, A\& A, 534, A23

Georgantopoulos, I., Comastri, A., Vignali, C., et al. 2013, A\& A, 555, A43

Gilli, R., Comastri, A., \& Hasinger, G. 2007, A\& $A$, 463, 79

Gilli, R., Vignali, C., Mignoli, M., et al. 2010, A\&A, 519, A92

Gilli, R., Su, J., Norman, C., et al. 2011, ApJ (Letters), 730, L28

Gilli, R. 2013, Mem. Soc. Astron. Italiana, 84, 647

Gofford, J., Reeves, J. N., Tombesi, F., et al. 2013, MNRAS, 430, 60

Harrison, C. M., Alexander, D. M., Swinbank, A. M., et al. 2012, MNRAS, 426, 1073

Heckman, T. M., Ptak, A., Hornschemeier, A., \& Kauffmann, G. 2005, ApJ, 634, 161

Hickox, R. C., Jones, C., Forman, W. R., et al. 2009, ApJ, 696, 891

Hopkins, P. F., Hernquist, L., Cox, T. J., \& Kereš, D. 2008, ApJS (Supplement), 175, 356

Houck, J. R., Soifer, B. T., Weedman, D., et al. 2005, ApJ (Letters), 622, L105 
Iwasawa, K., Crawford, C. S., Fabian, A. C., \& Wilman, R. J. 2005, MNRAS, 362, L20 Iwasawa, K., Gilli, R., Vignali, C., et al. 2012, A\& A, 546, A84

Lacy, M., Storrie-Lombardi, L. J., Sajina, A., et al. 2004, ApJS (Supplement), 154, 166

Lamastra, A., Menci, N., Fiore, F., et al. 2013, A $\mathscr{\xi} A$, 559, A56

Lanzuisi, G., Piconcelli, E., Fiore, F., et al. 2009, AछA, 498, 67

Maiolino, R., Gallerani, S., Neri, R., et al. 2012, MNRAS (Letters), 425, L66

Malizia, A., Stephen, J. B., Bassani, L., et al. 2009, MNRAS, 399, 944

Marconi, A., Risaliti, G., Gilli, R., et al. 2004, MNRAS, 351, 169

Martínez-Sansigre, A., Rawlings, S., Lacy, M., et al. 2005, Nature, 436, 666

Mateos, S., Alonso-Herrero, A., Carrera, F. J., et al. 2013, MNRAS, 434, 941

Menci, N., Fiore, F., Puccetti, S., \& Cavaliere, A. 2008, ApJ, 686, 219

Mignoli, M., Vignali, C., Gilli, R., et al. 2013, A\&A, 556, A29

Moretti, A., Vattakunnel, S., Tozzi, P., et al. 2012, A\&A, 548, A87

Mulchaey, J. S., Koratkar, A., Ward, M. J., et al. 1994, ApJ, 436, 586

Nesvadba, N. P. H., Lehnert, M. D., De Breuck, C., et al. 2008, A\&SA, 491, 407

Panessa, F., Bassani, L., Cappi, M., et al. 2006, A 8 A, 455, 173

Polletta, M. d. C., Wilkes, B. J., Siana, B., et al. 2006, ApJ, 642, 673

Ptak, A., Zakamska, N. L., Strauss, M. A., et al. 2006, ApJ, 637, 147

Ranalli, P., Comastri, A., Vignali, C., et al. 2013, A\&SA, 555, A42

Reeves, J. N., O'Brien, P. T., \& Ward, M. J. 2003, ApJ (Letters), 593, L65

Rodighiero, G., Daddi, E., Baronchelli, I., et al. 2011, ApJ (Letters), 739, L40

Rosario, D. J., Trakhtenbrot, B., Lutz, D., et al. 2013, A\&3A, 560, A72

Rupke, D. S. N. \& Veilleux, S. 2013, ApJ (Letters), 775, L15

Sacchi, N., La Franca, F., Feruglio, C., et al. 2009, ApJ, 703, 1778

Saez, C., Chartas, G., \& Brandt, W. N. 2009, ApJ, 697, 194

Sanders, D. B., Soifer, B. T., Elias, J. H., et al. 1988, ApJ, 325, 74

Sanders, D. B. \& Mirabel, I. F. 1996, ARA\& $A$, 34, 749

Severgnini, P., Caccianiga, A., Della Ceca, R., et al. 2011, A\& $A$, 525, A38

Severgnini, P., Caccianiga, A., \& Della Ceca, R. 2012, A\& $A$, 542, A46

Shi, Y., Helou, G., \& Armus, L. 2013, ApJ, 777, 6

Silk, J. \& Rees, M. J. 1998, A\&A (Letters), 331, L1

Soltan, A. 1982, MNRAS, 200, 115

Stern, D., Eisenhardt, P., Gorjian, V., et al. 2005, ApJ, 631, 163

Sturm, E., González-Alfonso, E., Veilleux, S., et al. 2011, ApJ (Letters), 733, L16

Tombesi, F., Cappi, M., Reeves, J. N., et al. 2010, A\&A, 521, A57

Tombesi, F., Cappi, M., Reeves, J. N., et al. 2011, ApJ, 742, 44

Tombesi, F., Cappi, M., Reeves, J. N., \& Braito, V. 2012, MNRAS (Letters), 422, L1

Tozzi, P., Gilli, R., Mainieri, V., et al. 2006, A\&A, 451, 457

Treister, E., Urry, C. M., \& Virani, S. 2009, ApJ, 696, 110

Treister, E., Natarajan, P., Sanders, D. B., et al. 2010, Science, 328, 600

Treister, E., Schawinski, K., Urry, C. M., \& Simmons, B. D. 2012, ApJ (Letters), 758, L39

Tueller, J., Mushotzky, R. F., Barthelmy, S., et al. 2008, ApJ, 681, 113

Vasudevan, R. V., Brandt, W. N., Mushotzky, R. F., et al. 2013, ApJ, 763, 111

Vignali, C., Alexander, D. M., \& Comastri, A. 2006, MNRAS, 373, 321

Vignali, C., Alexander, D. M., Gilli, R., \& Pozzi, F. 2010, MNRAS, 404, 48

Vignali, C., Mignoli, M., Gilli, R., et al., $A \xi A$, in preparation

Vito, F., Vignali, C., Gilli, R., et al. 2013, MNRAS, 428, 354

Weedman, D. W., Soifer, B. T., Hao, L., et al. 2006, ApJ, 651, 101

Winter, L. M., Lewis, K. T., Koss, M., et al. 2010, ApJ, 710, 503

Worsley, M. A., Fabian, A. C., Bauer, F. E., et al. 2005, MNRAS, 357, 1281

Xue, Y. Q., Luo, B., Brandt, W. N., et al. 2011, ApJS (Supplement), 195, 10

Xue, Y. Q., Wang, S. X., Brandt, W. N., et al. 2012, ApJ, 758, 129

Zakamska, N. L., Strauss, M. A., Krolik, J. H., et al. 2003, AJ, 126, 2125

Zubovas, K. \& King, A. R. 2012, MNRAS, 426, 2751 